\title{
Time-Slot Reservation based Multiple Access Protocol for the Internet of Things
}

\author{
ByungBog Lee ${ }^{1}$, Sang-Hyun Bae ${ }^{2}$, and Se-Jin Kim ${ }^{2 *}$ \\ ${ }^{1}$ IoT Research Division, Electronics and Telecommunications Research \\ Institute (ETRI), Daejeon 04520, Korea \\ ${ }^{2}$ Department of Computer Science and Statistics, Chosun University, \\ Gwangju 61452, Korea \\ bblee40@etri.re.kr, \{shbae,sjkim\}@chosun.ac.kr
}

\begin{abstract}
In the Internet of Things (IoT), a gateway and a set of IoT devices (IoTDs) form a startopology and the IoTDs compete between them for sending uplink communication during short time slots. Therefore, in this paper, we propose a novel multiple access protocol with time-slot reservation that can increase the success rate of the uplink data transmission with collision avoidance in the IoT. Further, using a modem calculator tool from the LoRa Alliance, we evaluate the system performance of the low power wide area network which is designed for sensors and applications in terms of equivalent bitrate, time on air, and Tx energy consumption for the uplink of the IoTDs.
\end{abstract}

Keywords: Internet of Things, Multiple Access Protocol, Time-Slot Reservation, Collision Avoidance

\section{Introduction}

Recently, the wireless sensor network (WSN) has become an essential part of many different fields, such as commercial [1-3], military [4-6], industrial [7-9], and medical areas [10-15], since they have significant advantages, i.e., low energy consumption, wireless distribution, and flexibility without cable restriction [16-18]. IEEE 802.15.4 standard was published in 2006 which defined the specifications of physical (PHY) and media access control (MAC) sublayers for low-rate wireless personal area networks, and it has become the de facto standard of WSNs [19]. The IEEE 802.15.4 standard uses a MAC protocol based on a carrier sense multiple access with collision avoidance (CSMA/CA) mechanism and networks can be built as either star or peer-to-peer topology [19].

In addition, the Internet of Things (IoT) is now receiving a great deal of attention from both academia and industry. The concept of the IoT is to emphasize ubiquitous computing among global networked machines and physical objects, denoted as things, such as sensors, actuators, machine-to-machine (M2M) devices, and IoT devices (IoTDs). Therefore, all the things surrounding us in our everyday lives will interact with each other and cooperate with other things [21]. Therefore, in the IoT, it is expected that a lot of IoTDs will be Internet-enabled using IEEE 802.15.4 PHY and MAC interfaces in various environments, e.g., home, buildings, and so on [21], [22]. However, one technology cannot serve all of the projected applications and volumes for the IoT. Thus, Wi-Fi and Bluetooth low energy (BLE) are widely adopted standards and serve the applications related to communicating personal devices quite well. Cellular technology is also a great fit for applications that need high data throughput and have a power source.

One of the important features for the IoT is the energy consumption. Low power wide area network (LPWAN) offers multi-year battery lifetime and is designed for

* Corresponding author. 
sensors and applications that need to send small amounts of data over long distances a few times per hour from varying environments [24-25]. In a LPWAN, the IoTDs are not associated with a specific gateway $(\mathrm{GW})$. Instead, data transmitted by an IoTD is typically received by multiple GWs. Each GW will forward the received packet from the IoTD to the cloud-based network server via some backhaul (either cellular, Ethernet, satellite, or Wi-Fi).

In this paper, we propose a novel multiple access protocol with time-slot reservation named time-slot reservation based multiple access protocol (TR-MAP) that can increase the success rate of the uplink data transmission with collision avoidance in the IoT [26]. We divide the procedure of the proposed TR-MAP into two stages. In stage 1, the IoTD performs frequency hopping and slot reserving as usual between retransmissions until retry counter doesn't exceed the number of the used channel frequencies, it opens receive window after each retransmission until the time slots have expired. In stage 2, the GW encodes the usable slot mask field to indicate the slots not used by IoTDs which uses the same frequency as the uplink. Further, using a modem calculator tool from the LoRa Alliance, we evaluate the system performance of the LPWAN which is designed for sensors and applications in terms of equivalent bitrate, time on air, and Tx energy consumption for the uplink of the IoTDs.

\section{Related Work}

\subsection{The Standard of IEEE 802.15.4 MAC Layers}

\section{A. IEEE 802.15.4-2003}

The MAC layer of the IEEE 802.15.4-2003 shows resemblance to the MAC layers of the WLAN IEEE 802.11 standards, in terms of the channel access strategies and the frame structures. Although carrier sense multiple access/collision avoidance (CSMA/CA) is the adopted channel access method, it however differs from the IEEE 802.11 standards as no Request to send/Clear to send (RTS/CTS) frames are employed. The MAC protocol of the standard supports two modes depending on whether beacons are enabled [20].

- $\quad$ Beacon-enabled: In this mode, periodic beacons are transmitted by the coordinate in order to maintain synchronization and exchange network information between the devices.

- Non Beacon-enabled: When the PAN operates in this modality, there are no beacons broadcasted by the coordinator. Access to channel in the network takes place using unslotted CSMA/CA mechanism. As noticed earlier, there are two versions of the CSMA/CA mechanism; slotted (beacon-enabled) and unslotted CSMA (non-beacon enabled).

\section{B. IEEE 802.15.4b-2006}

As stated earlier this version was released to remove ambiguities and clarify the original standard. In this regard, the use of the Guaranteed Time Slot was made optional, the scanning procedure was simplified. Furthermore the security suite usage was improved and more flexibility was introduced to the CSMA/CA procedure. With regard to the CSMA/CA mechanism; if the MAC layer evaluates that the CSMA/CA operations can not be completed in the current contention access period it waits for the next superframe and exercises another back off delay before evaluation unlike the 802.15.4-2003 case where this backoff delay was not applied before evaluation [19].

\subsection{Internet of Things}

Internet of Things (IoT) is a concept and a paradigm that considers pervasive presence in the environment of a variety of things/objects through wireless and wired connections. Further, each thing/object has a unique address to interact and cooperate with each other 
to create new applications/services and reach common goals. Figure 1 shows the concept of the IoT and an example of IoT applications. In Figure 1, an intelligent world with various smart environments that make energy, transport, cities and many other areas more intelligent. The goal of the IoT is to enable things to be connected anytime, anyplace, with anything and anyone by ideally using any path/network and any service [21-23].

\section{Internet of Things (IoT)}

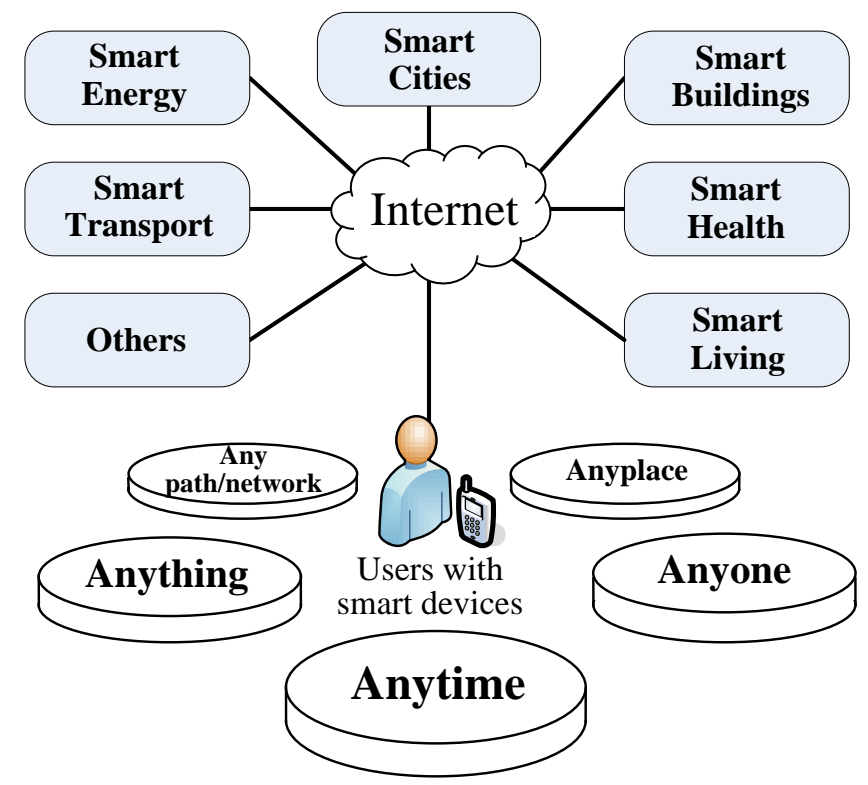

Figure 2. The Concept of Internet of Things

\section{Time-Slot Reservation based Multiple Access Protocol (TR-MAP)}

In this chapter, we propose the TR-MAP to reduce energy consumption of IoTDs. First, we introduce the slot timing model of the proposed TR-MAP to avoid systematic collisions or over-hearing problems. Then, we explain the proposed TR-MAP with two stages. The key parameters for the proposed TR-MAP MAC are listed in Table 1.

Table 1. Terminologies of the Proposed TR-MAP MAP

\begin{tabular}{|l|l|}
\hline Parameter & Description \\
\hline DevAddr & Device 32 bit network unicast or multicast address \\
\hline PushNb & $\begin{array}{l}\text { The number of opened push slots per beacon period. It must be a } \\
\text { power of 2 integer : PushNb }=2^{\mathrm{k}}\end{array}$ \\
\hline PushPeriod & $\begin{array}{l}\text { Period of the device receiver wake-up expressed in number of } \\
\text { slots : PushPeriod = (BeaconWindow / SlotLen) / PushNb }\end{array}$ \\
\hline PushOfiset & $\begin{array}{l}\text { Randomized offset computed at each push period start. The } \\
\text { range is from 0 to (PushPeriod-1) }\end{array}$ \\
\hline SlotLen & $\begin{array}{l}\text { Length of a unit push slot (push_data and push_ack transmition } \\
\text { time on air) }\end{array}$ \\
\hline NbPushSlot & $\begin{array}{l}\text { The beacon window interval is divided into } 2^{\mathrm{x}} \text { push slots of } \\
\text { SlotLen millisecond }\end{array}$ \\
\hline BeaconTime & $\begin{array}{l}\text { The time carried in the field (beacon payload). Time of the } \\
\text { immediately preceding beacon frame }\end{array}$ \\
\hline BeaconGuard & $\begin{array}{l}\text { The beacon frame time on air is actually much shorter than the } \\
\text { beacon reserved time interval to allow appending network }\end{array}$ \\
\hline
\end{tabular}




\begin{tabular}{|c|c|}
\hline & management broadcast frames in the future. \\
\hline Beacon Window & $\begin{array}{l}\text { The beacon window interval is divided into } 2^{\mathrm{x}} \text { from } 0 \text { to }\left(2^{\mathrm{x}}-1\right) \\
\text { slots of SlotLen : BeaconWindow }=\text { NbPushSlot }{ }^{*} \text { SlotLen }\end{array}$ \\
\hline BeaconPeriod & $\begin{array}{l}\text { The interval between the start of two successive beacons: } \\
\text { BeaconPeriod=BeaconGuard+BeaconReserved+BeaconWindow }\end{array}$ \\
\hline PushAckTimeoutCnt & $\begin{array}{l}\text { Acknowledgement timeout counter is incremented when an IoTD } \\
\text { pushes uplink frame and doesn't receive its ack. or downlink } \\
\text { frame within the time set by the downlink receive timeout delay }\end{array}$ \\
\hline MaxNbPush Retries & $\begin{array}{l}\text { The number of the maximum push retries should be equal or } \\
\text { more than the number of the frequencies (NbPushFreq) usable } \\
\text { for uplink access }\end{array}$ \\
\hline
\end{tabular}

\subsection{Slot Timing Model in the Proposed TR-MAP}

Consider the uplink of a LPWAN that consists of a GW and a set of IoTDs. We assume that the IoTDs are not associated with a specific GW. The GW is able to send and receive on different frequency channels at the time and is able to demodulate the signal without knowledge of the used spreading factor of the IoTD. The IoTD must open the send and receive slots at precise instants relative to the infrastructure beacon. Figure 2 illustrates the proposed TR-MAP timing model, the concept of beacon reception slot and data frame transmission slot (called "push slot") at a predictable time during a periodic time slot (the "Beacon Window" period is listed in the Table of Figure 2).

The beacon frame transmission is aligned with the beginning of the "Beacon Reserved" interval in Table 1. Each beacon is preceded by a guard time interval where no ping slot can be placed. The length of the guard interval corresponds to the time on air of the longest allowed frame. This is to insure that the data frame transmission initiated during a push slot just before the guard time will always have time to complete without colliding with the beacon transmission. The usable time interval for the push slot therefore spans from the end of the beacon reserved time interval to the beginning of the next beacon guard interval.

The IoTD also opens push slots every "Push Period". Most of the time these push slots are not used by the IoTD and therefore the IoTD reception window is closed or the radio transceiver is sleep as soon as the radio transceiver has assessed that no preamble is present on the radio channel or user data in not present. If uplink data has sent and then a preamble data is detected the radio transceiver will stay on until the downlink frame is demodulated. The MAC layer will then process the frame, check that its address field matches the IoTD address and that the message integrity check is valid before forwarding it to the application layer. 


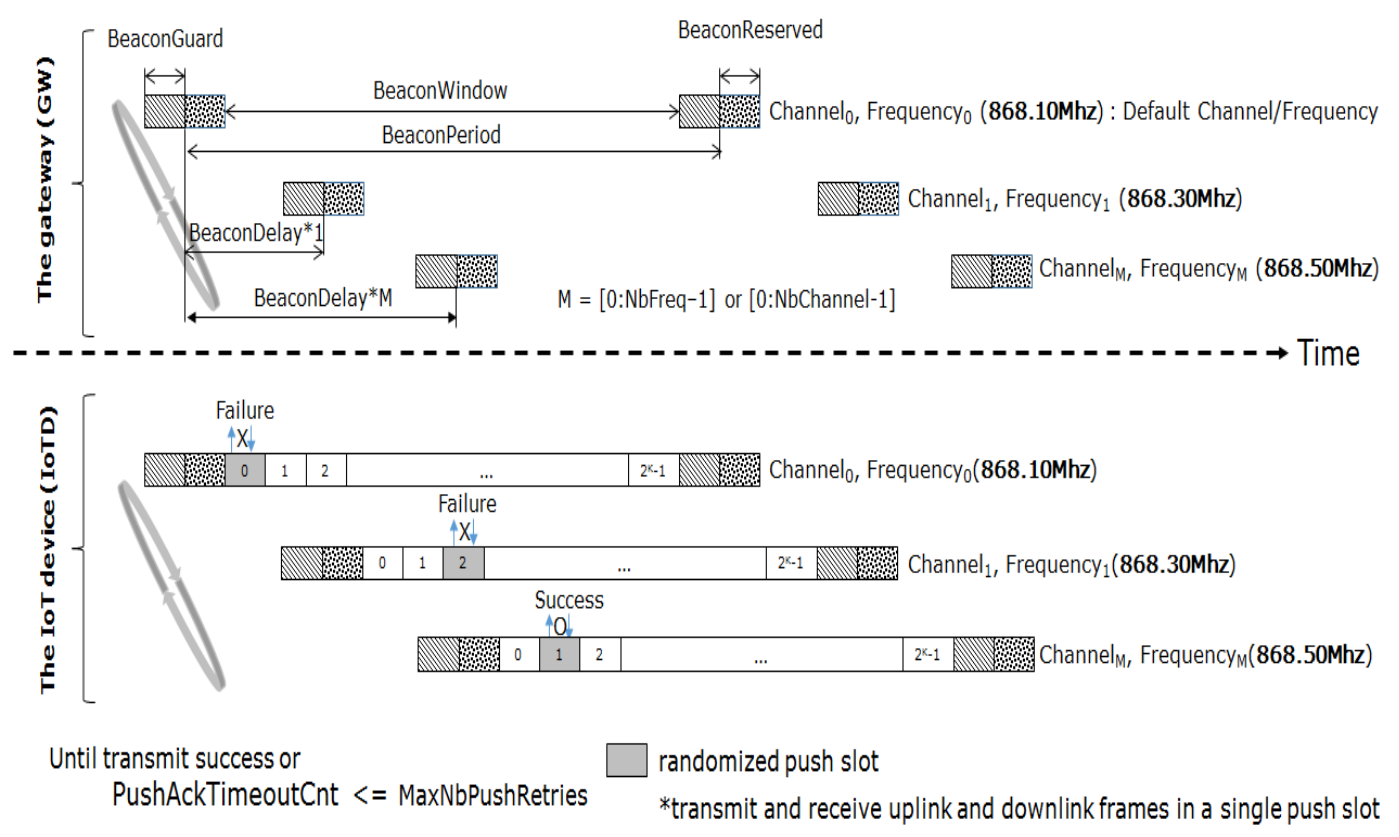

\begin{tabular}{|l|l|}
\hline BeaconGuard & $3000 \mathrm{~ms}$ \\
\hline BeaconReserved & $2120 \mathrm{~ms}$ \\
\hline BeaconWindow & NbPushSlot * SlotLen \\
\hline BeaconPeriod & BeaconGuard+BeaconReserved+BeaconWindow \\
\hline
\end{tabular}

Figure 2. The Beacon and Push Slots Timing.

\subsection{The Procedure of the Proposed TR-MAP}

Figure 3 shows the procedure of the proposed TR-MAP. To avoid systematic collisions or over-hearing problems, we divide the procedure of time slot reservation scheme into two stages.

In stage 1, the IoTD performs frequency hopping and slot reserving as usual between retransmissions until retry counter is not exceeded the number of the used channel frequencies, it opens receive window after each retransmission until the time slots have expired. The following explanation describes the slot reservation method that is used in the stage 1 .

The slot index is randomized and changed at every beacon period on each channel. The encryption scheme used is based on the generic algorithm described in IEEE 802.15.4/2006 Annex B [IEEE 802.15.4] using Advanced Encryption Standard (AES) with a key length of 128 bits. An AES encryption with a fixed key of all zeros is used to randomize:

$$
\text { Rand =aes128_encrypt }(\text { Key BeaconTime } \mid \text { DevAddr } \mid \text { pad16 })
$$

where Key is $16 \times 0 \times 00$. 


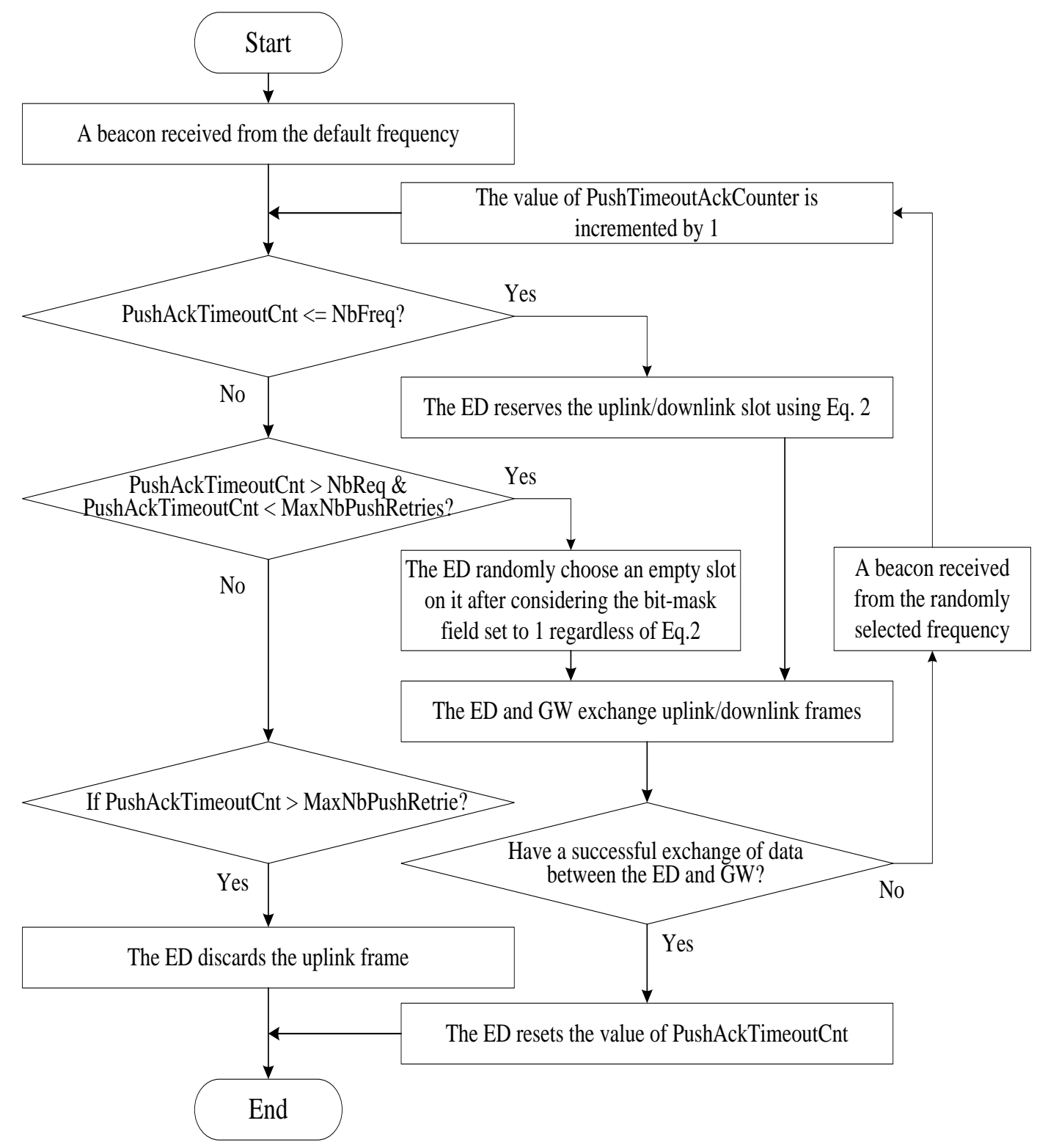

Figure 3. The Procedure of the Proposed TR-MAP

Then, we obtain the PushOffset, PO, can be expressed as

$$
\mathrm{PO}=(\operatorname{Rand}[0]+\operatorname{Rand}[1] \times 256) \% \mathrm{PP}
$$

where PP is PushPeriod. Further, the slots used for this beacon period will be:

$$
\mathrm{PO}+N \times \mathrm{PP} \text { with } N=[\mathrm{PushNb}-1]
$$

The IoTD manipulates slots starting at:

$$
\text { Slot } \mathrm{N}=\mathrm{BR}+(\mathrm{PO}+(N) \times \mathrm{PP}) \times \mathrm{SL}
$$

where $N=[0: \mathrm{PushNb}-1]$. In addition, BR and $\mathrm{SL}$ are BeaconReserved and SlotLen, respectively. 


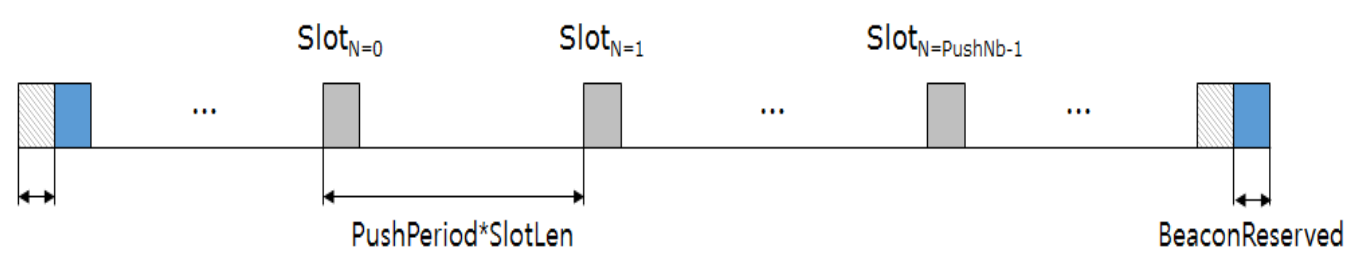

Figure 4. The Slots Used by the IoTD for this Beacon

Figure 4 describes the slots used by the IoTD in this beacon. If the IoTD did not succeed the data transmission in stage 1 , it performs the slot reservation and the data retransmission in stage 2 until the retry counter is equal to the retry counter limitation. If the retry counter exceeds the retry counter limitation, the data is never retransmitted and discarded.

The following explanation describes the slot reservation criteria that are used in stage 2. The acknowledgement timeout counter is incremented when an IoTD pushes uplink frame and doesn't receive its acknowledgement or downlink frame within the time set by the downlink receive timeout delay. The GW encodes the usable slot mask field to indicate the slots not used by IoTDs which use the same frequency as the uplink. A bit in the mask field set to 1 means that the corresponding slots can be used for the next uplink transmissions. The IoTD receives a beacon providing the mask field and randomly reserves one of slots in the mask field set to 1 .

\section{Simulation Results and Discussions}

In this section, we evaluate system performance of the proposed TR-MAP in terms of equivalent bitrate, time on air, and Tx energy consumption for the uplink of IoTDs using a modem calculator tool from the LoRa Alliance [24-25]. The centre frequency is $868 \mathrm{MHz}$ and we evaluate the system performance when the bandwidth (BW) is 125 and $250 \mathrm{kHz}$. Further, we use the spreading factor from 6 to 12 with coding rate 4/5. We assume that there are three payload length (PL), i.e., 10, 20, 30 bytes, and the transmit power is between $-2 \mathrm{dBm}$ to $20 \mathrm{dBm}$. The system parameters are listed in Table 2 .

Table 2. System Parameters

\begin{tabular}{|l|l|}
\hline Parameter & Value \\
\hline Centre frequency & $868 \mathrm{MHz}$ \\
\hline Bandwidth (BW) & $125 \mathrm{and} 250 \mathrm{kHz}$ \\
\hline Spreading factor & $6 \sim 12$ \\
\hline Coding rate & $4 / 5$ \\
\hline Payload Length (PL) & $10,20,30 \mathrm{bytes}$ \\
\hline Transmit power & $-2 \sim 20 \mathrm{dBm}$ \\
\hline
\end{tabular}




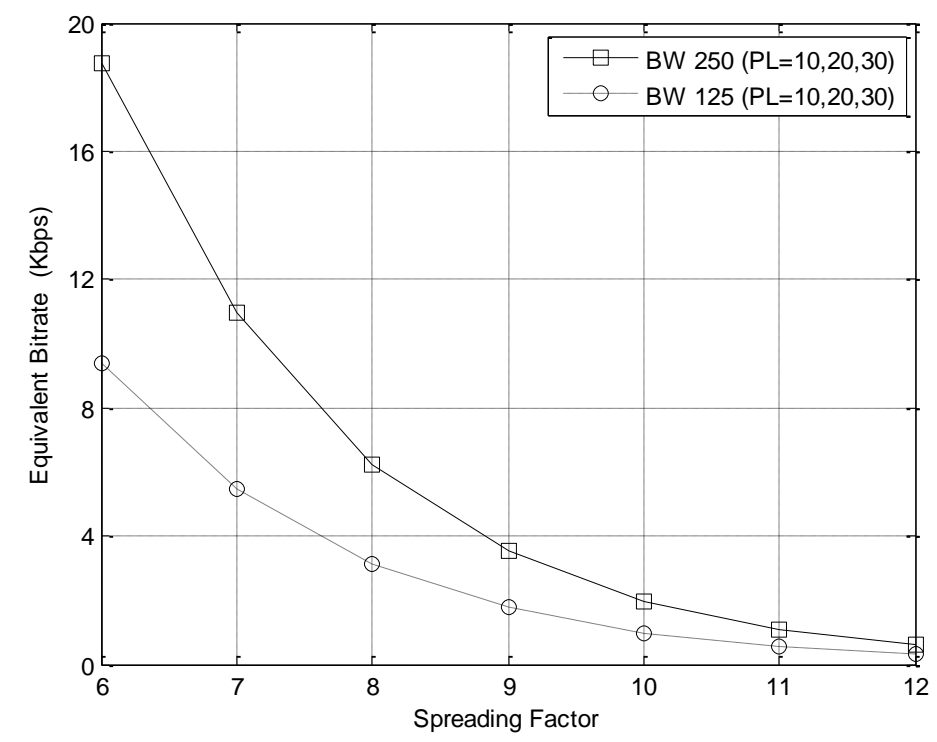

Figure 5. Equivalent Bitrate vs. Spreading Factor

Figure 5 depicts the results of the equivalent bitrate as the spreading factor increases when the BW is 125 and $250 \mathrm{kHz}$. It is shown that the results decrease as the spreading factor increases since the amount of data to transmit decreases. Further, the result with $\mathrm{BW} 250 \mathrm{kHz}$ is approximately twice bigger than the result with BW $125 \mathrm{kHz}$. We performed the simulation with different values of the payload size but results of the equivalent bitrate are the same when PL is 10, 20, and 30 in BW 125 and $250 \mathrm{kHz}$, respectively.

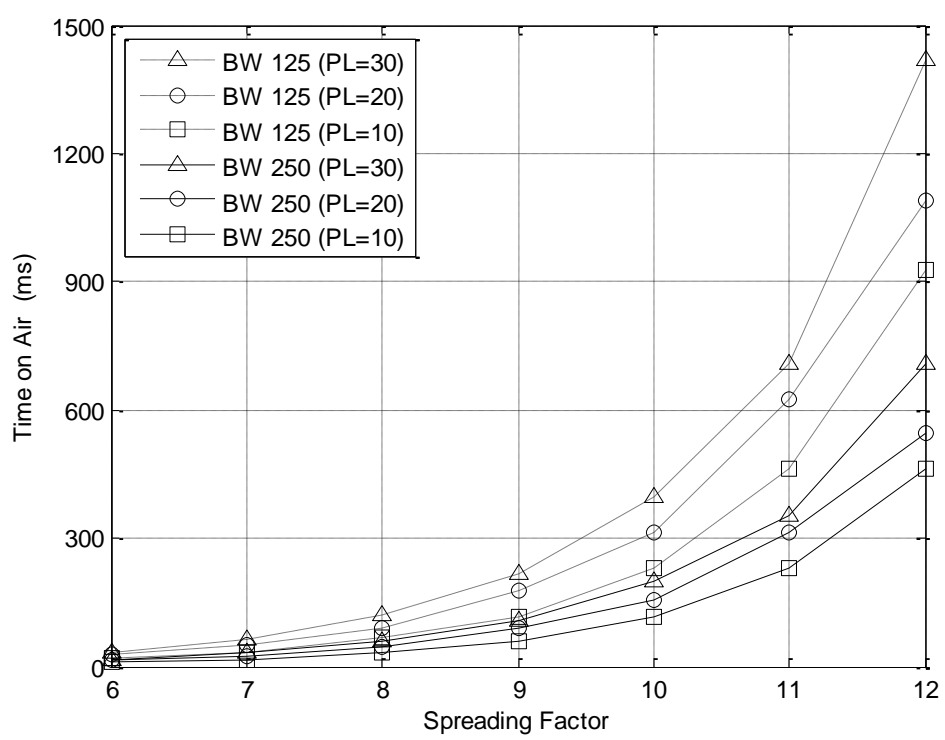

Figure 6. Time on Air vs. Spreading Factor

Figure 6 shows the results of the time on air in millisecond (ms) as the spreading factor increases when the $\mathrm{BW}$ is 125 and $250 \mathrm{kHz}$. It is shown that the results increase as the spreading factor increases since the transmit time increases. The results of $\mathrm{BW} 250 \mathrm{kHz}$ are lower than the results with $\mathrm{BW} 125 \mathrm{kHz}$. In addition. In 
each case of BW 125 and $250 \mathrm{kHz}$, the results are lower as the payload length decreases.

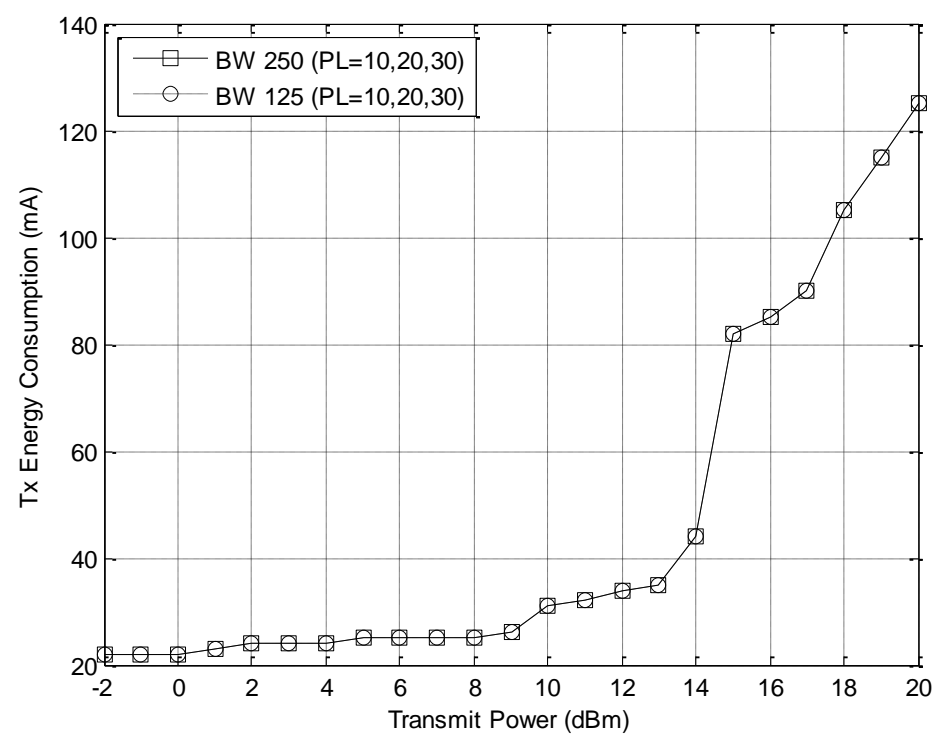

Figure 7. Tx Energy Consumption vs. Transmit Power

Figure 7 describes the results of the Tx energy consumption as the transmit power increases between -2 to $20 \mathrm{dBm}$ when the BW is 125 and $250 \mathrm{kHz}$. It is shown that the results are always the same when the payload length is 10,20 , and 30 with BW 125 and $250 \mathrm{kHz}$. This is because the Tx energy consumption is based on the transmit power regardless of the bandwidth and the payload length.

\section{Conclusions}

In this paper, we proposed a multiple access protocol with time-slot reservation that can increase the success rate of the uplink data transmission with collision avoidance in the IoT. The proposed TR-MAP has two stages. In stage 1, the IoTD performed frequency hopping and slot reserving as usual between retransmissions until retry counter doesn't exceed the number of the used channel frequencies, it opened receive window after each retransmission until the time slots have expired. In stage 2, the GW encoded the usable slot mask field to indicate the slots not used by IoTDs which uses the same frequency as the uplink. Further, using a modem calculator tool from the LoRa Alliance, we evaluated the system performance of the LPWAN which is designed for sensors and applications in terms of equivalent bitrate, time on air, and Tx energy consumption for the uplink of the IoTDs.

For future work, we plan to study an enhanced TR-MAP with multiple channels and sleep mode operations to further reduce the energy consumption of the IoT and WSNs.

\section{Acknowledgments}

This work was supported by the National Research Foundation of Korea (NRF) Grant funded by the Korean Government (MSIP) (No. 2015R1C1A1A01055869).

This paper is a revised and expanded version of a paper entitled two-stage time slot reservation multiple access scheme for communication collision avoidance presented at SUComS 2016, Amsterdam, Netherlands, 26-28 July, 2016. 


\section{References}

[1] A. Wheeler, E. Corporation, "Commercial Applications of Wireless Sensor Networks Using ZigBee," IEEE Communications Magazine, vol. 45, no. 4, pp. 70-77, (2007).

[2] V. Tsetsos, G. Alyfantis, T. Hasiotis, O. Sekkas and S. Hadjiefthymiades, "Commercial wireless sensor networks: technical and business issues", Second Annual Conference on Wireless On-demand Network Systems and Services, (2005), pp. 166-173.

[3] P. Lopez-Iturri, L. Azpilicueta, J. J. Astrain, E. Aguirre, E. Salinero, J. Villadangos and F. Falcone, "Implementation of Wireless Sensor Network Architecture for Interactive Shopping Carts to Enable Context-Aware Commercial Areas", IEEE Sensors Journal, vol. 16, no. 13, (2016), pp. 5416-5425.

[4] M. Winkler, K. D. Tuchs, K. Hughes and G. Barclay, "Theoretical and Practical aspects of military wireless sensor networks", Journal of Telecommunications and Information Technology, (2008), pp. 3745.

[5] S. Lee, S. Lee, H. Song and H. Lee, "Wireless sensor network design for tactical military applications: Remote large-scale environments", MILCOM 2009, (2009), pp. 1-7.

[6] M. P. Đurišić, Z. Tafa, G. Dimić, V. Milutinović, "A survey of military applications of wireless sensor networks", Mediterranean Conference on Embedded Computing (MECO) 2012, (2012), pp. 196-199.

[7] A. Willig, K. Matheus and A. Wolisz, "Wireless Technology in Industrial Networks", Proceedings of the IEEE, vol. 93, no. 6, (2005), pp. 1130-1151.

[8] M. Bal, "Industrial applications of collaborative Wireless Sensor Networks: A survey", International Symposium on Industrial Electronics (ISIE) 2014, (2014), pp. 1463-1468.

[9] X. Shen, Z. Wang and Y. Sun, "Wireless sensor networks for industrial applications", Intelligent Control and Automation, 2004, vol. 4, (2004), pp. 3636-3640.

[10] J. Ko, C. Lu, M. B. Srivastava, J. A. Stankovic, A. Terzis and M. Welsh, "Wireless Sensor Networks for Healthcare", Proceedings of the IEEE, vol. 98, no. 11, (2010), pp. 1947-1960.

[11] X. Zhang, H. Jiang, L. Zhang, C. Zhang, Z. Wang and X. Chen, "An Energy-Efficient ASIC for Wireless Body Sensor Networks in Medical Applications", IEEE Transactions on Biomedical Circuits and Systems, vol. 4, no. 1, (2010), pp. 11-18.

[12] J. Pan, S. Li and Z. Xu, "Security mechanism for a wireless-sensor-network based healthcare monitoring system", IET Communications, vol. 6, no. 18, (2012), pp. 3274-3280.

[13] J. A. Gutierrez, M. Naeve, E. Callaway, M. Bourgeois, V. Mitter and B. Heile, "IEEE 802.15.4: a developing standard for low-power low-cost wireless personal area networks", IEEE Network, vol. 15, no. 5, (2001), pp. 12-19.

[14] K. Lorincz, D. J. Malan, T. R. F. Fulford-Jones, A. Nawoj, A. Clavel, V. Shnayder, G. Mainland, M. Welsh and S. Moulton, "Sensor networks for emergency response: challenges and opportunities", IEEE Pervasive Computing, vol. 3, no. 4, (2004), pp. 16-23.

[15] K. M. S. Thotahewa, J. Redouté and M. R. Yuce, "A Low-Power Wearable Dual-Band Wireless Body Area Network System: Development and Experimental Evaluation”, IEEE Transactions on Microwave Theory and Techniques, vol. 62, no. 11, (2014), pp. 2802-2811.

[16] K. Sohraby, D. Minoli and T. Znati, "Wireless Sensor Networks: Technology, Protocols, and Applications", Wiley, (2007).

[17] W. Akyildiz, E. Y. Su and E. Cayirci, “A survey on sensor networks”, IEEE Communications Magazine, vol. 40 no. 8, (2002), pp. 102-114, 2002.

[18] C. Chong, S. Kumar, "Sensor networks: evolution, opportunities, and challenges", Proceedings of the IEEE, vol. 91, no. 8, (2003), pp. 1247-1256.

[19] IEEE 802.15.4-2006, IEEE Standard for Information Technology - Part 15.4: Wireless Medium Access Control (MAC) and Physical Layer (PHY) Specification for Low Rate Wireless Personal Area Networks (LR-WPANs), (2006).

[20] IEEE 802.15.4-2003, IEEE Standard for Information Technology - Part 15.4: Wireless Medium Access Control (MAC) and Physical Layer (PHY) Specification for Low Rate Wireless Personal Area Networks (LR-WPANs), (2003).

[21] D. Giusto, A. Iera, G. Morabito, L. Atzori, The Internet of Things, Springer, (2010)

[22] C. Perera, A. Zaslavsky, P. Christen and D. Georgakopoulos, "Context Aware Computing for The Internet of Things: A Survey", IEEE Communications Surveys \& Tutorials, vol. 16, no. 1, (2014), pp. 414-454.

[23] B. Lee and S. Kim, "Energy-Efficient Sensor Device Personalization Scheme for the Internet of Things and Wireless Sensor Networks", IEICE Trans. on Communications, vol. E98-B, no. 1, (2015), pp. 231241.

[24] LoRa Alliance, "This Whitepaper is sponsored by the LoRa Alliance Members, and in particular, our Platinum Sponsors of the November 2015 All Member Meeting", (2015).

[25] LoRa Alliance, https://www.lora-alliance.org.

[26] B. Lee, B. Choi and S. Kim, "Two-Stage Time Slot Reservation Multiple Access Scheme for Communication Collision Avoidance", Advanced Science and Technology Letters, vol. 137 (SUComS 2016), (2016), pp. 171-176. 


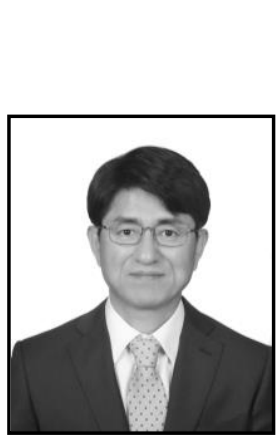

\begin{abstract}
Authors
ByungBog Lee, he received his B.S., M.S. and Ph.D. degrees in Computer Science from Howon University, Chonbuk National University and Korea University in 1991, 1993 and 2010, respectively. He is currently a principal researcher at the Electronics and Telecommunications Research Institute, Korea. His current research interests include mobile communications, sensor networks, machine-to-machine networks, and Internet-of-Things networks. He is currently doing researches into the smartphone-based thing personalization schemes and thing finder/browser for Internet-ofThings networks.
\end{abstract}

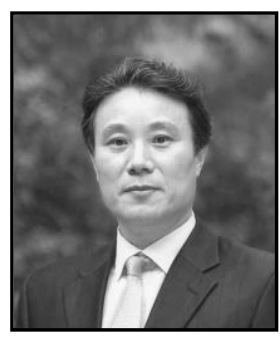

Sang-Hyun Bae, he received the B.S. and M.S. degrees from the Department of Electrical Engineering at Chosun University, Gwangju, Korea, in 1982 and 1984, and Ph.D. degree from the Department of Information Science at Tokyo Metropolitan University, Tokyo, Japan, in 1988, respectively. He was a researcher in the Department of Electrical Engineering at Tokyo Institute of Technology, Japan in 1985. Also, he was a visiting professor in the Department of Information Engineering at Nara Institute of Technology, Japan in 1997 and the Department of Information Engineering at University of Alberta, Canada in 2002 and a member of board of directors of NRF, Korea in 2012-2013. He is a professor in the Department of Computer Science and Statistics at Chosun University, Gwangju, Korea.

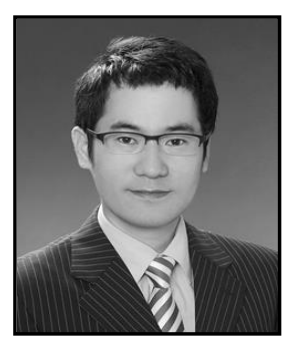

Se-Jin kim, he received the B.S. degree from the Department of Computer Science at Chosun University, Gwangju, Korea, in 2004, and M.S. and Ph.D. degrees from the Department of Computer Science at Korea University, Seoul, Korea, in 2006 and 2010, respectively. He was a post-doctoral fellow in the Department of Electrical Engineering at University of Washington, Seattle, USA and the Department of Electrical and Computer Engineering at University of British Columbia, Vancouver, Canada in 2012 and 2013-2014, respectively. He is currently an assistant professor in the Department of Computer Science and Statistics at Chosun University, Gwangju, Korea. His research interests cover a wide range of topics in broadband wired and wireless communication networks such as wireless resource management, interference management, and so on for the next generation mobile communication network. 
International Journal of Future Generation Communication and Networking Vol. 9, No.8, (2016) 